Research Paper

\title{
Effects of Notch2 and Notch3 on Cell Proliferation and Apoptosis of Trophoblast Cell Lines
}

\author{
Wei-Xiu Zhao, Xu Zhuang, Tao-Tao Huang, Ran Feng, and Jian-Hua Lin ${ }^{\bowtie}$ \\ Department of Obstetrics and Gynecology, Ren Ji Hospital, School of Medicine, Shanghai Jiao Tong University, Shanghai, China \\ $\square$ Corresponding author: Jian-Hua Lin, Department of Obstetrics and Gynecology, Ren Ji Hospital, School of Medicine, Shanghai Jiao Tong University, \\ Shanghai, P.R.China. Email: linjhuarj@126.com
}

() 2015 Ivyspring International Publisher. Reproduction is permitted for personal, noncommercial use, provided that the article is in whole, unmodified, and properly cited. See http:/ /ivyspring.com/terms for terms and conditions.

Received: 2015.06.11; Accepted: 2015.09.20; Published: 2015.10.20

\begin{abstract}
Aims: To investigate the effect of Notch2 and Notch3 on cell proliferation and apoptosis of two trophoblast cell lines, BeWo and JAR.

Methods: Notch2 and Notch3 expression in BeWo and JAR cells was upregulated or downregulated using lentivirus-mediated overexpression or RNA interference. The effect of Notch2 and Notch 3 on cell proliferation was assessed by the CCK-8 assay. The effect of Notch2 and Notch3 on the apoptosis of BeWo and JAR cells was evaluated by flow cytometry using the Annexin V-PE Apoptosis kit. Lentivirus-based overexpression vectors were constructed by cloning the full-length coding sequences of human Notch2 and Notch3 C-terminally tagged with GFP or GFP alone (control) into a lentivirus-based expression vector. Lentivirus-based gene silencing vectors were prepared by cloning small interfering sequences targeting human Notch2 and Notch3 and scrambled control RNA sequence into a lentivirus-based gene knockdown vector. The effect of Notch2 and Notch3 on cell proliferation was assessed by the CCK-8 assay. And the effect of Notch2 and Notch3 on the apoptosis of BeWo and JAR cells was evaluated by flow cytometry using the Annexin V PE Apoptosis kit.

Results: We found that the downregulation of Notch2 and Notch3 gene expression in BeWo and JAR cells resulted in an increase in cell proliferation, while upregulation of Notch3 and Notch2 expression led to a decrease in cell proliferation. Moreover, the overexpression of Notch3 and Notch2 in BeWo and JAR cells reduced apoptosis in these trophoblast cell lines, whereas apoptosis was increased in the cells in which the expression of Notch3 and Notch2 was downregulated.

Conclusions: Notch2 and Notch3 inhibited both cell proliferation and cell apoptosis in BeWo and JAR trophoblast cell lines.
\end{abstract}

Key words: BeWo, JAR, Notch2, Notch3, proliferation, apoptosis

\section{Introduction}

Notch signaling is an evolutionarily conserved pathway. It plays important roles in many cellular processes, such as cell fate determination and cell differentiation [1]. Four Notch receptors (Notch1-4) and 5 ligands (Jag1 and 2 and Dll1, 3, and 4) are found in the mammalian $[2,3]$. As a receptor, Notch can be bound by a ligand which in turn activates Notch signaling in the canonical pathways [4].

Our previous study shows a dramatic increase in Notch3 expression and a significant decrease in Notch2 expression in placentas with early-onset severe preeclampsia compared to those in normal pla- centas [5]. Therefore, Notch signaling pathway may play a role in the pathogenesis of preeclampsia. Till now, however, a potential role for Notch signaling in preeclampsia has not been elucidated.

Trophoblasts can perform multiple cellular behaviors, such as differentiation, proliferation, apoptosis, migration and invasion (interstitial invasion and endovascular invasion), which are important for the development of normal placenta. The placenta is arguably the most important transient organ of the body. Placental dysfunction is associated with pregnancy complications [6]. Abnormal trophoblast pro- 
liferation, invasion and apoptosis have been identified in placentas with preeclampsia [7-12]. Our previous work demonstrates that Notch2 and Notch3 are mainly expressed by villous cytotrophoblasts [5]. Therefore, Notch2, Notch3 may exert effects on cytotrophoblast functions. To date, few studies have been carried out to address the potential effect of Notch2 and Notch3 on trophoblast proliferation and apoptosis. So in this study we used choriocarcinoma cell lines, BeWo and JAR, to determine if Notch2 and Notch3 affect trophoblast proliferation and apoptosis. The results showed that Notch2 and Notch3 exerted effects on cell proliferation and apoptosis of the two trophoblast cell lines.

\section{Materials and Methods}

\section{Lentivirus vector construction}

The lentivirus vectors for lentivirus-mediated upregulation and downregulation of Notch2 and Notch3 expression were purchased from GenePharma Co., Ltd (Shanghai, China). To construct lentivirus-mediated overexpression of Notch2 and Notch3, the full-length coding sequences of human Notch2 and Notch3 C-terminally tagged with GFP or GFP alone (control) were cloned into the lentivirus vector with empty vector lentivirus as negative control known as Notch-NC. To generate lentivirus-mediated silencing vector, small interfering (5'-GCAGGTA GCTCAGACCATTCT-3') sequence targeting human Notch2 and scrambled control RNA sequence (5'TTCTCCGAACGTGTCACGT-3'), named as shNotch2-NT, were cloned into the lentivirus knockdown vector. Similarly, to produce lentivirus-mediated silencing system targeting human Notch3, small interfering (5'-ATCTCCAGCATTACT ACCGAG-3') sequence and scrambled control RNA sequence ( $5^{\prime}$-TTCTCCGAACGTGTCACG $-3^{\prime}$ ), named as shNotch3-NT, were cloned into the lentivirus knockdown vector.

\section{Cell culture and transfection}

Human choriocarcinoma cell lines, BeWo and JAR, were from American Type Culture Collection (ATCC, Manassas, VA, USA). Cells were cultured in DMEM Medium (Life Technologies, Carlsbad, CA, USA) supplemented with $10 \%$ heat inactivated fetal bovine serum (FBS) (Life Technologies, Carlsbad, CA, USA), 1\% Pen/Strep (Life Technologies, Carlsbad, CA, USA) and grown at $37^{\circ} \mathrm{C}$ in a $5 \% \mathrm{CO} 2$ incubator. In this study, Notch3-overexpression, Notch2 shRNA encoding lentiviruses and their control lentiviruses were used to infect BeWo cells. Notch2-overexpression, Notch3 shRNA encoding lentiviruses and their control lentiviruses were used to infect JAR cells. Fluorescence microscopy and quantitative real-time PCR (qRT-PCR) assay were used to monitor infection efficiency when infected cells were collected $48 \mathrm{~h}$ and $72 \mathrm{~h}$ respectively post lentivirus infection.

\section{RNA extraction, reverse transcription and qRT-PCR}

Infected cells were collected and subjected to RNA extraction, reverse transcription and qRT-PCR. Total RNA was isolated with TRIzol reagent (Life technologies, Carlsbad, California). RNA purity, yield, and integrity were determined spectrophotometrically. Reverse transcription was performed using FastQuant RT Kit (TIANGEN, Beijing, China) following manufacturer's instructions. RT-PCR was performed for Notch2 and Notch3 genes with the ABI 7500 detection system (Applied Biosystems, Foster City, California) using SuperReal PreMix Plus (TIANGEN, Beijing, China). The total reaction was carried out in a $20 \mu \mathrm{L}$ system under the following conditions: denaturation at $95^{\circ} \mathrm{C}$ for $15 \mathrm{~min}$, followed by 40 cycles of $95{ }^{\circ} \mathrm{C}$ for $10 \mathrm{sec}, 60{ }^{\circ} \mathrm{C}$ for $32 \mathrm{sec}$. Primers for Notch2 and Notch3 genes were listed in Table 1. Negative controls were performed without cDNA in the reaction mixture. The results were normalized against glyceraldehyde-3-phosphate dehydrogenase (GAPDH) gene expression. The relative quantification of target gene was performed with standard curve or comparative cycle threshold (CT) method.

Table 1. Notch2 and Notch3 Primer Pairs for RT-PCR Analysis.

\begin{tabular}{ll}
\hline Gene & Primers (5'-3') \\
\hline Notch2 & F-TCAACTGCCAAGCGGATGT \\
Notch3 & R-CTTGGCTGCTTCATAGCTCC \\
& F-GCTCAACGGCACTGATCCT \\
GAPDH & R-AGCCCAGTGTAAGGCTGATT \\
& F-CGGAGTCAACGGATTTGGTCGTATTGG \\
& R-GCTCCTGGAAGATGGTGATGGGATTTCC \\
\hline
\end{tabular}

\section{Cell proliferation assay}

Cell proliferation was assessed by the Cell Counting Kit8 (CCK-8) (Dojindo Laboratories, Kumamoto, Japan). Briefly, cells were harvested $24 \mathrm{~h}$ after infection by lentivirus. Then the infected cells were seeded on 96-well microplate at a density of 3 $\times 10^{3}$ cells per well. The cells were cultured for $24 \mathrm{~h}, 48$ $\mathrm{h}$ and $72 \mathrm{~h}$. Then, $10 \mu \mathrm{L}$ of CCK-8 solution was added to each well, and incubated at $37^{\circ} \mathrm{C}$ for an additional 3 $\mathrm{h}$. Optical density (OD) was determined at a wave-length of $450 \mathrm{~nm}$.

\section{Apoptosis analysis}

The effect of Notch2 and Notch3 on the apoptosis of BeWo and JAR cells was evaluated by flow cytometry using the Annexin V PE Apoptosis kit (BD 
Pharmingen, USA). Firstly, BeWo and JAR cells were infected with lentivirus and treated with EDTA-free trypsin at $72 \mathrm{~h}$ culture time. Afterwards, cells were washed by $1 \times \mathrm{PBS}\left(4^{\circ} \mathrm{C}\right)$, followed by resuspending the cell pellet with $300 \mu \mathrm{L}$ of $1 \times$ Binding Buffer. Next, $5 \mu \mathrm{l}$ of Annexin V-PE were added to the cell suspension for $15 \mathrm{~min}$ in the dark at room temperature, according to the manufacturer's instructions. Five $\mu$ l of 7-AAD solution was added in the cell suspension $5 \mathrm{~min}$ before flow cytometry analysis and then $200 \mu \mathrm{l}$ of $1 \times$ Binding Buffer was added for flow cytometry analysis. The percentage of apoptotic cells was evaluated by FACS Calibur (BD Biosciences, USA).

\section{Statistical analysis}

The data were presented as means \pm SD of the experiments. Statistical significance was assessed with Student's t-test and the difference was considered significant when P-value was less than 0.05 .

\section{Results}

Generation of BeWo and JAR cells with altered Notch2 and Notch3 expression by lentivirus-based
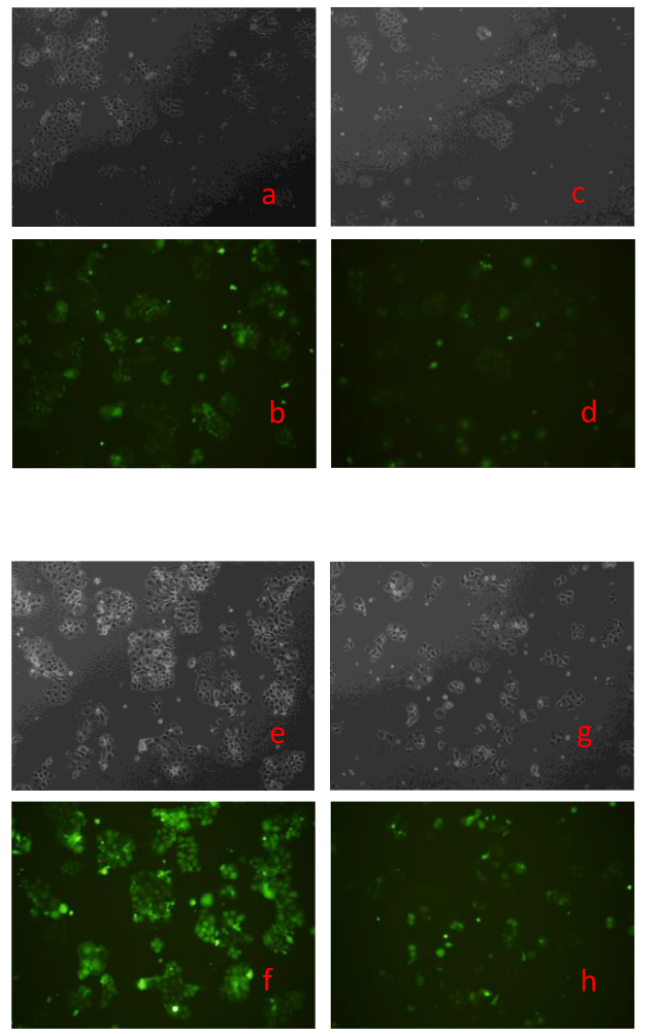

approaches.

Our previous results showed that BeWo cells express higher levels of Notch2 but lower levels of Notch3 than JAR cells. Therefore, in this study, we used the following four types of cells derived from BeWo or JAR cell lines to investigate the effect of Notch2 and Notch3 on trophoblast proliferation and apoptosis: a) BeWo cells in which Notch 2 expression was knocked down; b) BeWo cells in which Notch3 was overexpressed; c) JAR cells in which Notch2 was overexpressed; and d) JAR cells in which Notch3 expression was knocked down.

To generate these cells, BeWo cells were infected with lentiviruses expressing Notch2 shRNA or Notch3 cDNA, while JAR cells were infected with lentiviruses expressing Notch2 cDNA or Notch3 shRNA. After $48 \mathrm{~h}$, infection efficiency was assessed with fluorescence microscope. As shown in Fig. 1 and Fig. 2, the GFP tag in lentiviruses detected infection efficiency in bright (Fig. 1a, c, e, g and Fig. 2a, c, e, g) and fluorescence (Fig. 1b, d, f, h, and Fig. 2b, d, f, h, ) microscope.
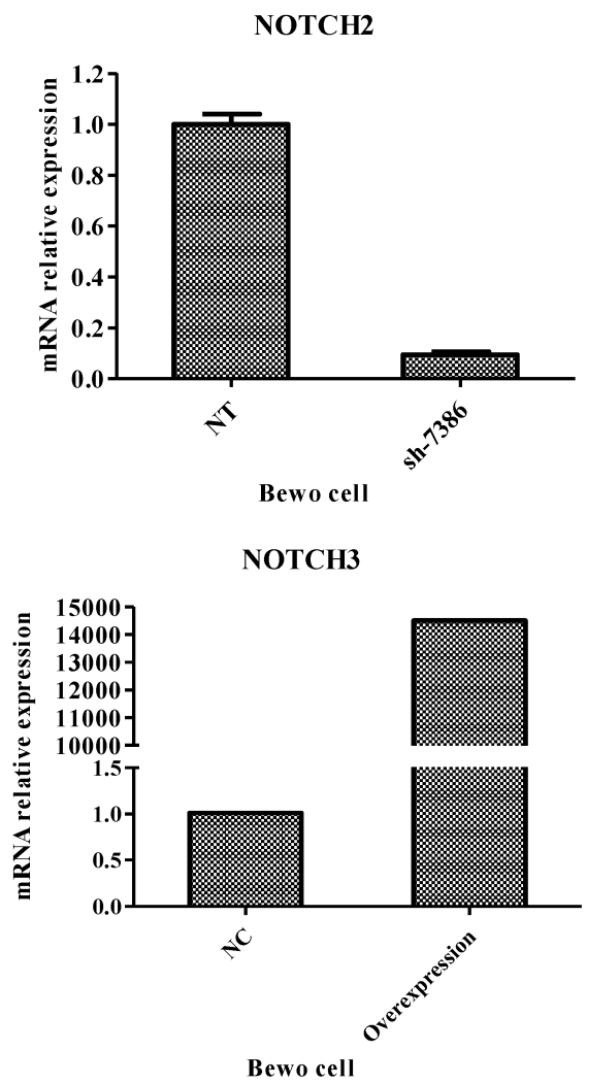

Fig. 1. Transfection efficiency at $48 \mathrm{~h}$ after Notch2 shRNA and Notch3-overexpression lentivirus transfection to BeWo cells. Fig. 1: Green fluorescent protein (GFP) expression was detected by a fluorescence microscope and mRNA expression of BeWo cells was assessed by qRT-PCR method. The lentiviral vectors carried GFP gene. Therefore, the BeWo cells infected by Notch-carrying lentivirus with GFP expression can be identified with a fluorescence microscope at $48 \mathrm{~h}$ after infection. Cell morphology of the BeWo cells infected with Notch2 shRNA and Notch3-overexpression lentivirus under a microscope was shown in Fig. 1c and Fig. 1g. GFP expression of BeWo cells infected with Notch2 shRNA and Notch3-overexpression lentivirus was detected by a fluorescence microscope (Fig. 1d and Fig. 1h). Cell morphology of the BeWo cells infected with shNotch2-NT and Notch3-NC lentivirus under a microscope was shown in Fig. 1a and Fig. le. GFP expression of BeWo cells infected with shNotch2-NT and Notch3-NC lentivirus was identified by a fluorescence microscope (Fig. $1 \mathrm{~b}$ and Fig. 1f). As shown in Fig. 1, transfection efficiency was $>90 \%$. As shown in the right panel of Fig. 1 , mRNA expression of BeWo cells infected by Notch2 shRNA lentivirus was significantly decreased compared with that of BeWo cells infected by shNotch2-NT lentivirus, while that of BeWo cells infected by Notch3-overexpression lentivirus was significantly increased compared with control. 

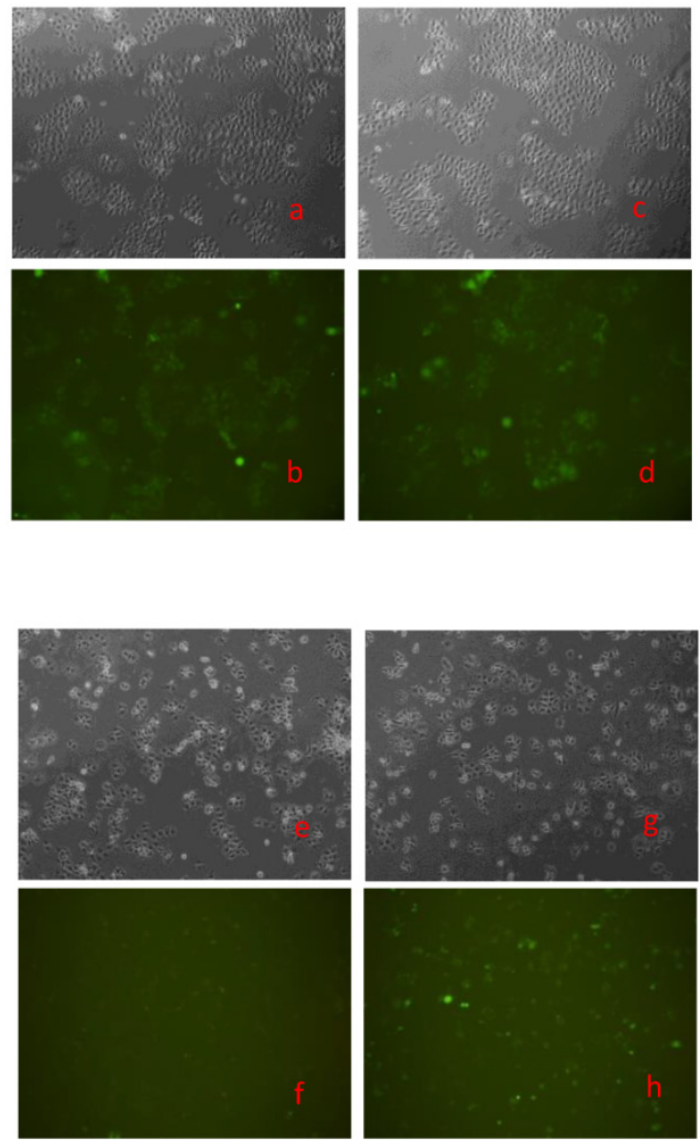

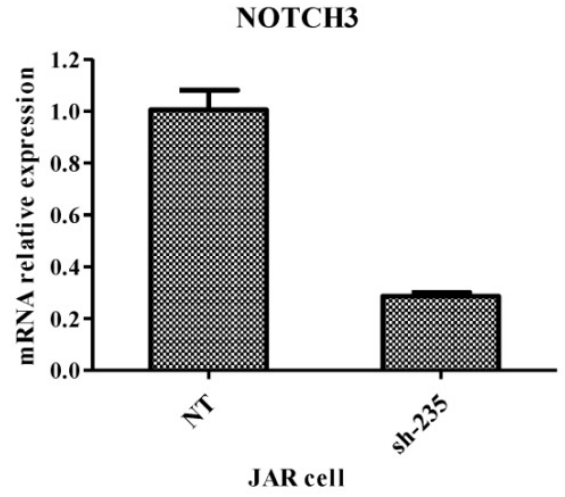

NOTCH2

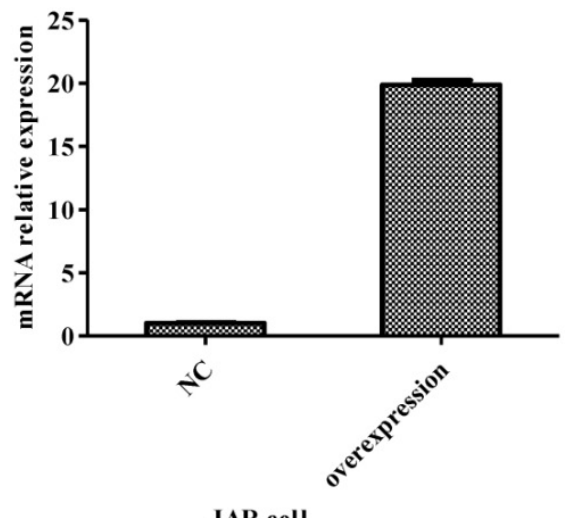

Fig. 2. Transfection efficiency at $48 \mathrm{~h}$ after Notch 3 shRNA and Notch2-overexpression lentivirus transfection to JAR cells. Fig. 2: Green fluorescent protein (GFP) expression was detected by a fluorescence microscope and mRNA expression of JAR cells was assessed byqRT-PCR method. The lentiviral vectors carried GFP gene. Therefore, the JAR cells infected by Notch-carrying lentivirus with GFP expression can be identified with a fluorescence microscope at $48 \mathrm{~h}$ after transfection. Cell morphology of the JAR cells infected with Notch3 shRNA and Notch2-overexpression lentivirus under a microscope was shown in Fig. 2c and Fig. $2 \mathrm{~g}$. GFP expression of JAR cells infected with Notch3 shRNA and Notch2-overexpression lentivirus was detected by a fluorescence microscope (Fig. $2 \mathrm{~d}$ and Fig. 2h). Cell morphology of the JAR cells infected with shNotch3-NT and Notch2-NC lentivirus under a microscope was shown in Fig. 2a and Fig. 2e. GFP expression of JAR cells infected with shNotch3-NT and Notch2-NC lentivirus was identified by a fluorescence microscope (Fig. 2b and Fig. 2f). As shown in Fig. 2, transfection efficiency was $>80 \%$. As shown in the right panel of Fig. 2, mRNA expression of JAR cells infected by Notch 3 shRNA lentivirus was significantly decreased compared with that of JAR cells infected by shNotch3-NT lentivirus, whereas that of JAR cells infected by Notch2-overexpression lentivirus was significantly increased compared with control.

Both in BeWo and in JAR cells, $>80 \%$ cells were infected successfully. To determine the change of Notch expression, Notch mRNA was analyzed by RT-PCR. Notch mRNA was decreased more than $60 \%$ after Notch shRNA lentivirus infection when compared to control-shRNA (the right panel of Fig. 1 and Fig. 2). Notch mRNA expression after Notch-overexpressing lentivirus infection was much higher than control (the right panel of Fig. 1 and Fig. 2 ). Therefore, these data indicated that we were able to obtain BeWo and JAR cells in which Notch proteins were either overexpressed or downregulated significantly enough to carry out functional assays.

\section{Notch2 and Notch3 inhibit proliferation of BeWo and JAR cells}

The effect of Notch 2 and Notch3 on cell proliferation was assessed by the CCK-8 assay. It was found that downregulation of Notch2 gene expression in
BeWo cells increased BeWo cell proliferation (the upper panel of Fig. 3, $\mathrm{P}<0.05$ ), and upregulation of Notch3 gene expression inhibited cell proliferation starting $48 \mathrm{~h}$ after Notch3-overexpressing lentivirus infection (the lower panel of Fig. 3, $\mathrm{P}<0.05$ ). There was no significant change in cell proliferation at $24 \mathrm{~h}$ after Notch3-overexpressing lentivirus infection when compared with control (the lower panel of Fig. 3, $P>0.05$ ).

JAR cell proliferation was enhanced after Notch3 shRNA lentivirus infection (the upper panel of Fig. 4, $\mathrm{P}<0.05$ ), while cell growth rate of the Notch2-overexpressing lentivirus infection group was significantly lower than that of the control group (the lower panel of Fig. 4, $\mathrm{P}<0.01$ ) except that no significant difference was shown between two groups after $24 \mathrm{~h}$ of culture (the lower panel of Fig. 4, P>0.05). 

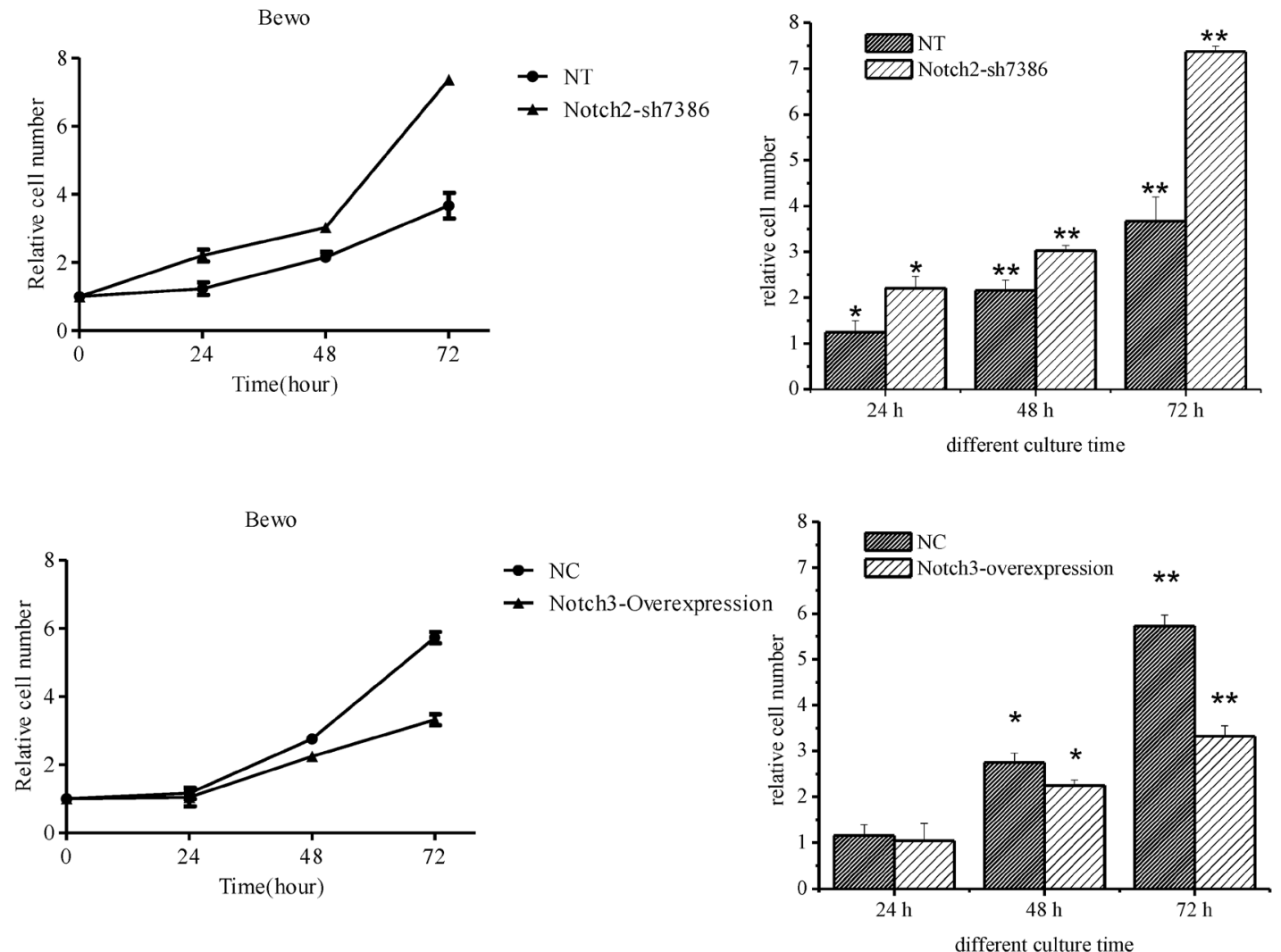

Fig. 3. Effect of Notch2 downregulation and Notch3 overexpression on proliferation of BeWo cells. Fig. 3: Downregulation of Notch2 gene expression in BeWo cells resulted in enhancement of proliferation. Upregulation of Notch 3 expression in BeWo cells inhibited cell proliferation. NT: negative control test, NC: negative control, * P<0.05, ** $\mathrm{P}<0.01$.
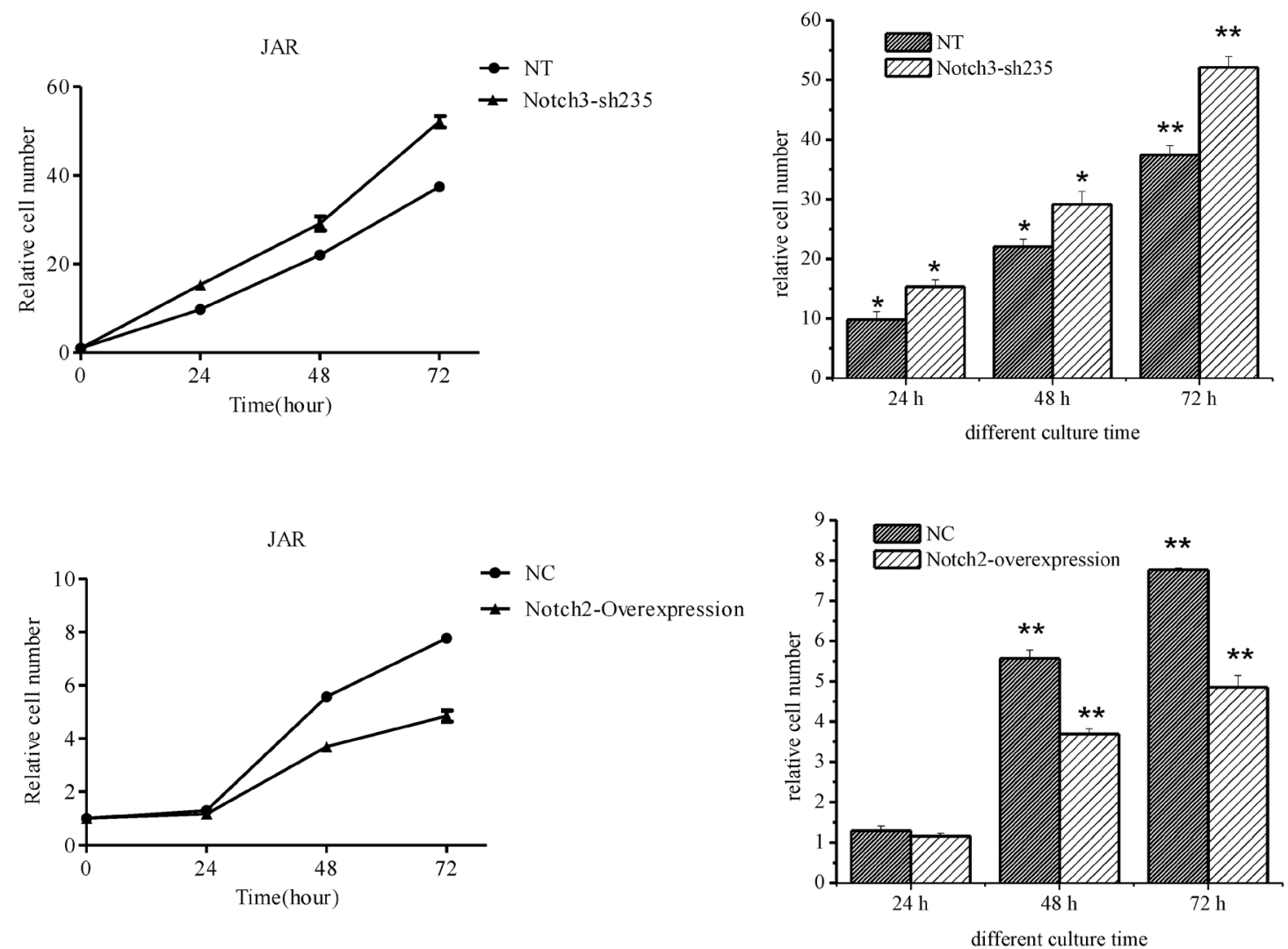

Fig. 4. Effect of Notch 3 downregulation and Notch 2 overexpression on proliferation of JAR cells. Fig. 4: Downregulation of Notch 3 expression in JAR cells enhanced cell proliferation. Upregulation of Notch2 expression in JAR cells suppressed cell proliferation. NT: negative control test, NC: negative control, ${ }^{*}$ P<0.05, ** P<0.01. 


\section{Notch2 and Notch3 inhibit apoptosis of BeWo and JAR cells}

The percentage of apoptotic cells was determined by flow cytometry after Annexin V/PE and 7-AAD staining. Annexin V-PE only positive cells in the lower right quadrant of the FL2/FL3 dot plot were considered to be early apoptotic cells and those labeled with annexin V-PE and 7-AAD in the upper right quadrant were late apoptotic cells. The results showed that apoptosis of BeWo cells was inhibited
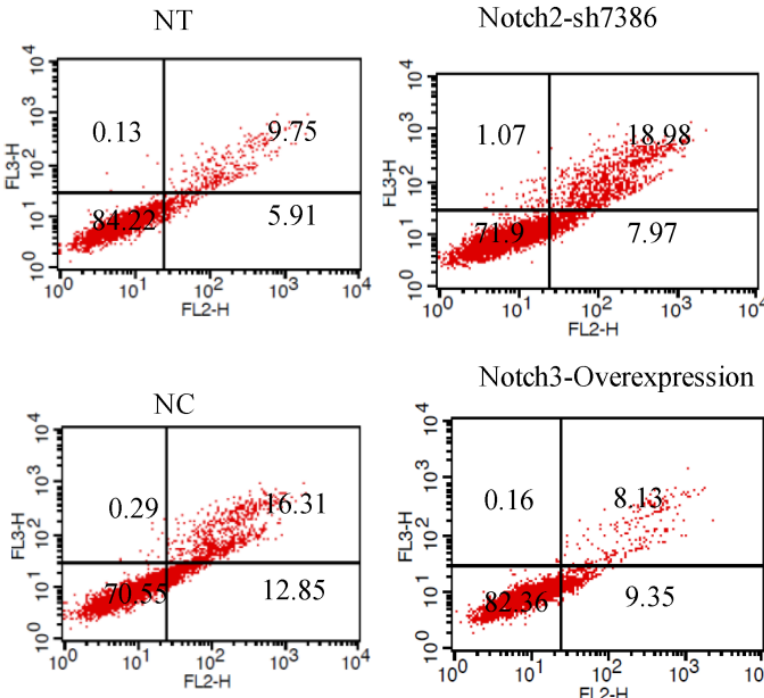

Notch3-Overexpression
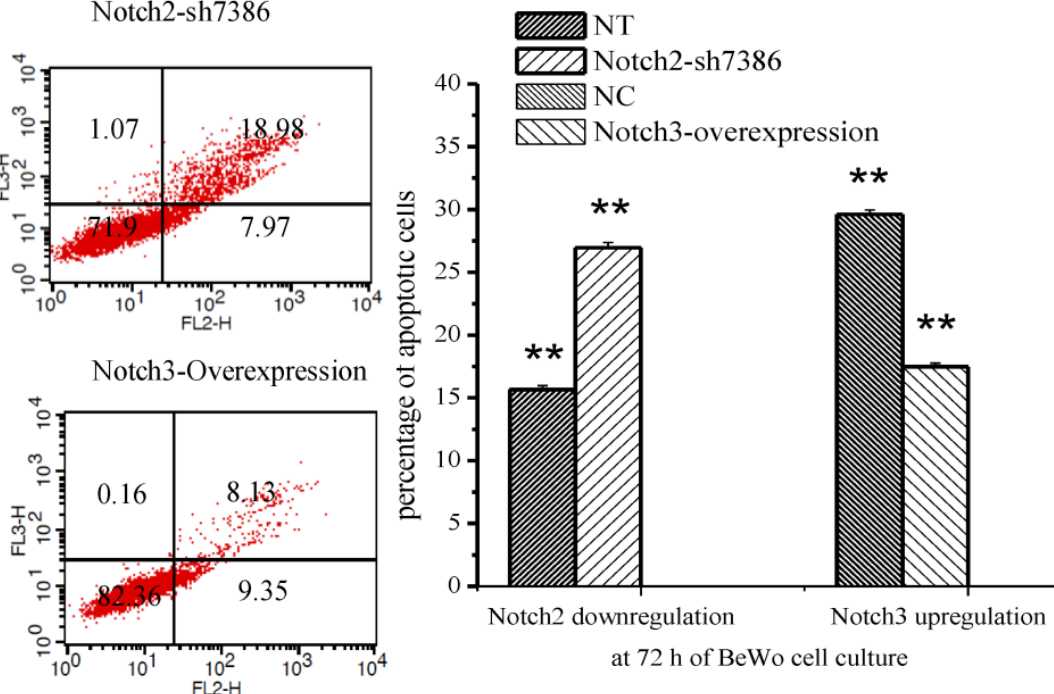

at $72 \mathrm{~h}$ of BeWo cell culture

Fig. 5. Effect of Notch2 and Notch3 on apoptosis of BeWo cells. Fig. 5: The different cells can be identified: viable cells with Annexin V-/7-AAD- in the lower left quadrant, already dead cells with Annexin V-/7-AAD+ in the upper left quadrant, late apoptotic cells with Annexin V+/7-AAD+ in the upper right and early apoptotic cells with Annexin $\mathrm{V}+/ 7-\mathrm{AAD}-$ in the lower right quadrants. The percentage of late and early apoptotic cells were summed to give the total number of apoptotic cells. The results demonstrated that apoptosis of BeWo cells was inhibited after Notch3-overexpressing lentivirus infection (Fig. $5, \mathrm{P}<0.01$ ), whereas the apoptotic cells were significantly increased at $72 \mathrm{~h}$ after Notch2 shRNA lentivirus infection (Fig. 5, P<0.01). NT: negative control test, NC: negative control, ** $P<0.01$.

$72 \mathrm{~h}$
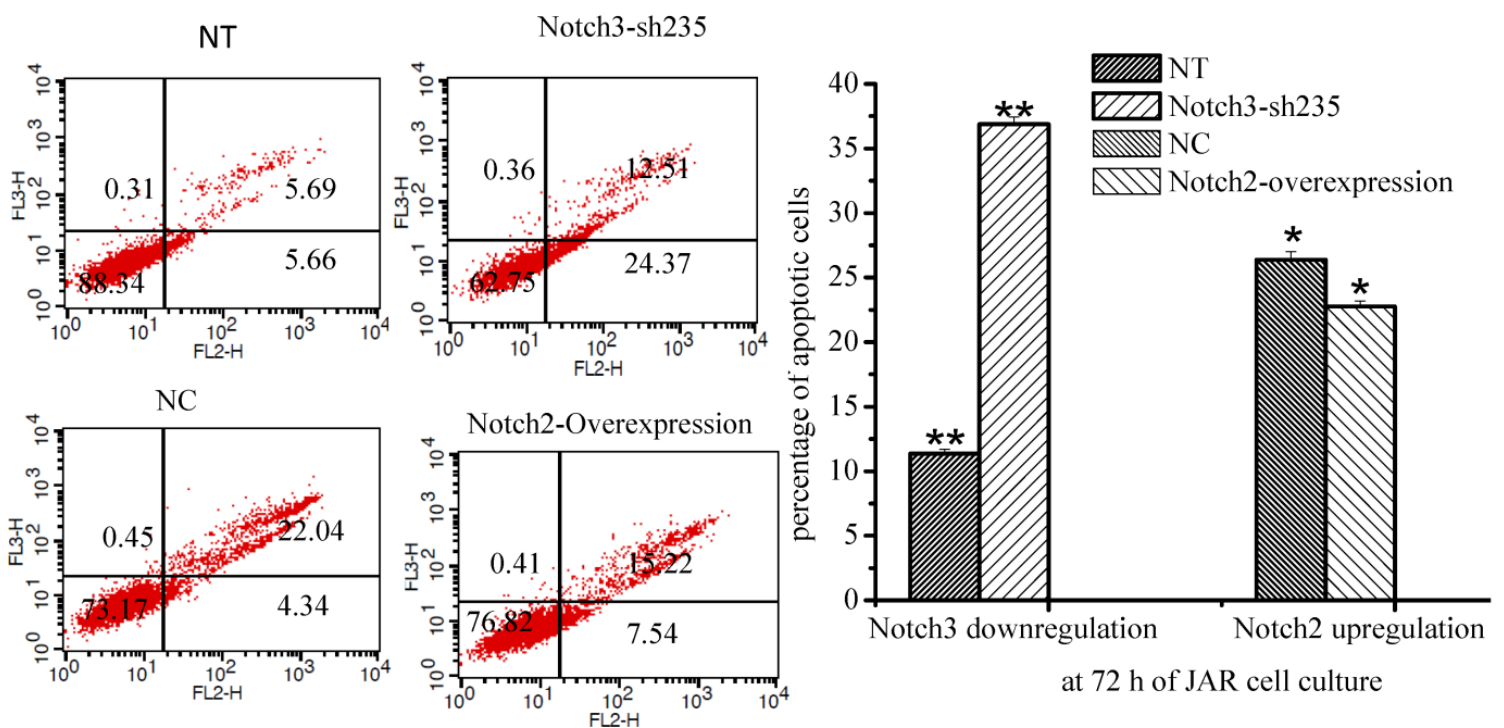

at $72 \mathrm{~h}$ of JAR cell culture

Fig. 6. Effect of Notch2 and Notch3 on apoptosis of JAR cells. Fig. 6: The different cells can be identified: viable cells with Annexin V-/7-AAD- in the lower left quadrant, already dead cells with Annexin V-/7-AAD+ in the upper left quadrant, late apoptotic cells with Annexin V+/7-AAD+ in the upper right and early apoptotic cells with Annexin $\mathrm{V}+/ 7-A A D-$ in the lower right quadrants. The percentage of late and early apoptotic cells were summed to give the total number of apoptotic cells. As shown in Fig. 6 , apoptosis of JAR cell was promoted after downregulation of Notch3 expression $(P<0.01)$, and it was inhibited at $72 \mathrm{~h}$ after Notch2-overexpressing lentivirus infection ( $<<0.05)$. NT: negative control test, NC: negative control, $* \mathrm{P}<0.05$, $* * \mathrm{P}<0.01$. 


\section{Discussion}

Our studies demonstrated that Notch2 and Notch3 affected proliferation and apoptosis of choriocarcinoma cell lines, BeWo and JAR. Downregulation of Notch2 expression in BeWo and Notch3 expression in JAR cells promoted cell proliferation and enhanced cell apoptosis, while upregulation of Notch3 and Notch2 expression resulted in suppression of cell proliferation and protection from apoptosis of cells. These results indicated that Notch2 and Notch3 played a role in trophoblast cell proliferation and apoptosis.

The present study demonstrated Notch2 and Notch3 regulated cytotrophoblast cell proliferation. Trophoblast, including cytotrophoblast, is the important component of villi. Villous cytotrophoblast cells grow throughout gestation. Proliferation of cytotrophoblast cells and differentiation into syncytiotrophoblast are essential elements of the growth in villous surface area [13]. Mayhew and Simpson showed that villous trophoblasts are kept in a tightly-regulated steady state with the relative amounts of cytotrophoblasts and syncytiotrophoblasts or relative numbers of nuclei $[14,15]$. Therefore, abnormal proliferation of cytotrophoblasts may perturb this steady state and result in complicated pregnancies, such as preeclampsia. Some studies have found that preeclampsia is complicated by abnormal proliferation. Arnholdt found that trophoblast proliferation rate in preeclampsia is increased when compared with that in normal pregnancy [16]. Another paper also showed an excess of proliferative immature intermediate trophoblasts in preeclampsia placentas [17]. However, the study from Prusac demonstrated that there is more proliferation in placentas complicated with HELLP syndrome, not preeclampsia, compared with placentas of normal gestation [11]. These inconsistent results may result from different gestational age of the samples and different study methods.

Given that normal proliferation of trophoblasts plays important roles in pregnancy, it is essential to elucidate the underlying mechanism for proliferation of trophoblasts. Haider has provided evidence that Notch signaling could be a key regulatory pathway of controlling trophoblast proliferation and found that cell proliferation in the different primary trophoblast model systems is elevated following chemical inhibition of Notch signaling [18]. Wagener showed that Notch1 can regulate proliferation of JEG3 cells through p21 or ERK1/ 2 pathway [19]. Our previous study has demonstrated that Notch2 and Notch3 are mainly expressed by villous cytotrophoblast cells [5], however, few studies have been carried out to address the potential effect of Notch2 and Notch3 on trophoblast proliferation so far [20], including BeWo and JAR cells. Plessl found that Notch2 does not change proliferation of trophoblasts [20], whereas our present study showed that downregulation of Notch2 and Notch3 expression can promote proliferation of BeWo and JAR cells, which was in agreement with the result from Haider [18]. We found that overexpression of Notch 2 and Notch3 can inhibit proliferation of BeWo and JAR cells. The difference between our result and that of Plessl may be attributed to different materials (placental tissue versus cell lines) and methods. Plessl analysed proliferation in placental villous explant cultures and primary cytotrophoblasts by specific antibodies blocking Notch2 pathway, while cell proliferation of trophoblast cell lines was assessed using lentivirus-mediated overexpression or RNA interference of Notch in this study.

It is well known that proliferation and apoptosis are important in life cycle of normal trophoblast cells. In the normal human placenta, trophoblast apoptotosis is associated with trophoblast differentiation [21]. Huppertz et al demonstrated that initiation stage of apoptosis cascade for formation of syncytiotrophoblast must be confined to cytotrophoblast, a population of proliferating cells and then new cellular material for formation of syncytiotrophoblast layer can be continuously provided [22]. Given that apoptosis of cytotrophoblast is well correlated with proliferation, we hypothesized that effects of Notch 2 and Notch 3 on cytotrophoblast proliferation will be reflected in cell apoptosis as well.

This present study also showed that Notch2 and Notch3 regulate apoptosis of cytotrophoblast cells. They protected BeWo and JAR cells from apoptosis. It has been demonstrated that apoptosis takes place within placental tissue and increases significantly as pregnancy progresses, which suggested that apoptosis may exert an effect on the normal development of placentas [23]. Apoptotic changes can occur in both cytotrophoblast and syncytiotrophoblast of human placental villi [13]. Abnormal apoptosis in placenta may play a role in complicated pregnancy. Smith found increased apoptosis in placentas complicated by intrauterine growth restriction [24]. There is more placenta apoptosis in preeclampsia than in normal pregnancy $[11,12,25]$.

Many factors are involved in apoptosis process, such as Fas, Fas ligand (Fas-L), caspase-8, Bcl-2 family, Mcl-1、p53 [26]. There are few reports on the effect of Notch signaling pathway on apoptosis of cytotrophoblasts [27], and there is no study about the role of Notch2 and Notch 3 in apoptosis of cytotrophoblasts till now. Notch 2 and Notch3 which are mainly expressed in villous cytotrophoblast cells [5] can regu- 
late proliferation of BeWo and JAR cells in the present paper, and since villous trophoblasts are kept in a steady state with the relative amounts of cytotrophoblast and syncytiotrophoblast [14, 15], we hypothesize that Notch2 and Notch3 may take part in cytotrophoblast apoptosis. Consistent with this hypothesis, our result showed that overexpression of Notch3 and Notch2 inhibited apoptosis of BeWo and JAR cells, which are cellular models for human cytotrophoblasts.

However, these results about proliferation and apoptosis of BeWo and JAR cells were inconsistent with our previous study which showed that Notch2 expression is decreased, whereas Notch3 expression is increased in placentas complicated by preeclampsia compared with normal pregnancy [5]. Perhaps this inconsistency can be explained by compensatory increase in Notch3 expression in response to decrease of Notch2 expression in placentas complicated by preeclampsia.

There are some limitations in this study. First, the main of this study is represented by the exclusive use of two tumor cell lines, the choriocarcinoma cell lines BeWo and JAR. These are tumor cell lines that might respond differently from primary trophoblast cells although they have often been used as models for studying villous trophoblast behaviour. The further study is needed to confirm the results in primary trophoblast cells. Second, the molecular pathways involved in regulating proliferation and apoptosis of BeWo and JAR cells by Notch have not been investigated in the present study.

Overall, the present study demonstrated that Notch2 and Notch3 regulated proliferation and apoptosis of BeWo and JAR cells. Given that abnormal expression of Notch2 and Notch3 in placentas with preeclampsia, it is suggested that Notch2 and Notch3 may be involved in the pathogenesis of preeclampsia through their effect on cytotrophoblast proliferation and apoptosis. Further studies are required to elucidate the potential roles of Notch2 and Notch3 in the other functions of cytotrophoblasts, such as invasion and differentiation, which are important for normal placental development.

\section{Acknowledgment}

The authors would like to thank $\mathrm{Xu}$ Feng for kind advice and careful edition for the present study.

This work was supported by the National Natural Science Foundation of China, No. 81170589/H0420, Grants from Shanghai Health Bureau Key Disciplines and Specialties Foundation, Shanghai Education Commission Key Disciplines Foundation, Key Discipline Project of Renji Hospital, Shanghai Jiaotong University School of Medicine.

\section{Competing Interests}

The authors declared no potential conflicts of interest with respect to the research, authorship, and/or publication of this article.

\section{References}

1. Bray SJ. Notch signalling: a simple pathway becomes complex. Nat Rev Mol Cell Biol. 2006;7(9):678-689.

2. Kopan R, Ilagan MX. The canonical Notch signaling pathway: unfolding the activation mechanism. Cell. 2009;137(2):216-233.

3. D'Souza B, Meloty-Kapella L, Weinmaster G. Canonical and non-canonical Notch ligands. Curr Top Dev Biol. 2010;92:73-129.

4. Pan D, Rubin GM. Kuzbanian controls proteolytic processing of Notch and mediates lateral inhibition during Drosophila and vertebrate neurogenesis. Cell. 1997;90(2):271-280.

5. Zhao WX, Huang TT, Jiang $M$, et al. Expression of notch family proteins in placentas from patients with early-onset severe preeclampsia. Reprod Sci. 2014;21(6):716-723

6. Burton GJ, Fowden AL. The placenta: a multifaceted, transient organ. Philos Trans R Soc Lond B Biol Sci. 2015;370(1663):20140066.

7. Ray JE, Garcia J, Jurisicova A, et al. Mtd/Bok takes a swing: proapoptotic Mtd/Bok regulates trophoblast cell proliferation during human placental development and in preeclampsia. Cell Death Differ. 2010;17(5):846-859.

8. Staribratova D, Zaprianov Z, Milchev N. [Proliferation of villous trophoblast and stroma in normal and pathologic pregnancies (preeclampsia)]. Akush Ginekol (Sofiia). 2005;44(2):20-22.

9. Zhou Y, Damsky CH, Chiu K, et al. Preeclampsia is associated with abnormal expression of adhesion molecules by invasive cytotrophoblasts. J Clin Invest. 1993;91(3):950-960.

10. McMaster MT, Zhou Y, Fisher SJ. Abnormal placentation and the syndrome of preeclampsia. Semin Nephrol. 2004;24(6):540-547.

11. Prusac IK, Zekic Tomas S, Roje D. Apoptosis, proliferation and Fas ligand expression in placental trophoblast from pregnancies complicated by HELLP syndrome or pre-eclampsia. Acta Obstet Gynecol Scand. 2011;90(10):1157-1163.

12. Tomas SZ, Prusac IK, Roje D, et al. Trophoblast apoptosis in placentas from pregnancies complicated by preeclampsia. Gynecol Obstet Invest. 2011;71(4):250-255

13. Mayhew TM. Turnover of human villous trophoblast in normal pregnancy: what do we know and what do we need to know? Placenta. 2014;35(4):229-240.

14. Mayhew TM, Simpson RA. Quantitative evidence for the spatial dispersal of trophoblast nuclei in human placental villi during gestation. Placenta. 1994;15(8):837-844

15. Simpson RA, Mayhew TM, Barnes PR. From 13 weeks to term, the trophoblast of human placenta grows by the continuous recruitment of new proliferative units: a study of nuclear number using the disector. Placenta. 1992;13(5):501-512.

16. Arnholdt H, Meisel F, Fandrey K, et al. Proliferation of villous trophoblast of the human placenta in normal and abnormal pregnancies. Virchows Arch B Cell Pathol Incl Mol Pathol. 1991;60(6):365-372.

17. Redline RW, Patterson P. Pre-eclampsia is associated with an excess of proliferative immature intermediate trophoblast. Hum Pathol. 1995;26(6):594-600.

18. Haider S, Meinhardt G, Velicky P, et al. Notch signaling plays a critical role in motility and differentiation of human first-trimester cytotrophoblasts. Endocrinology. 2014;155(1):263-274.

19. Wagener J, Yang W, Kazuschke K, et al. CCN3 regulates proliferation and migration properties in Jeg3 trophoblast cells via ERK1/2, Akt and Notch signalling. Mol Hum Reprod. 2013;19(4):237-249.

20. Plessl K, Haider S, Fiala C, et al. Expression pattern and function of Notch2 in different subtypes of first trimester cytotrophoblast. Placenta. 2015;36(4):365-371.

21. Aires MB, Dos Santos AC. Effects of maternal diabetes on trophoblast cells. World J Diabetes. 2015;6(2):338-344.

22. Huppertz B, Kadyrov M, Kingdom JC. Apoptosis and its role in the trophoblast. Am J Obstet Gynecol. 2006;195(1):29-39.

23. Smith SC, Baker PN, Symonds EM. Placental apoptosis in normal human pregnancy. Am J Obstet Gynecol. 1997;177(1):57-65.

24. Smith SC, Baker PN, Symonds EM. Increased placental apoptosis in intrauterine growth restriction. Am J Obstet Gynecol. 1997;177(6):1395-1401.

25. Sharp AN, Heazell AE, Baczyk D, et al. Preeclampsia is associated with alterations in the p53-pathway in villous trophoblast. PLoS One. 2014:9(1):e87621.

26. Heazell AE, Crocker IP. Live and let die - regulation of villous trophoblast apoptosis in normal and abnormal pregnancies. Placenta. 2008;29(9):772-783.

27. Tan T, Lu B, Zhang J, et al. Notch1 signaling antagonizes transforming growth factor-beta pathway and induces apoptosis in rabbit trophoblast stem cells. Stem Cells Dev. 2014;23(8):813-822. 